\title{
Reason Code
}

National Cancer Institute

\section{Source}

National Cancer Institute. Reason Code. NCI Thesaurus. Code C93643.

A coded value specifying the rationale for an activity or entity. 